\title{
GEFÄSSE MIT SCHRÄGEN UND SENKRECHTEN PLASTISCHEN LEISTEN IM FUNDMATERIAL DER SPÄTEN PHASE DER TARNOBRZEG LAUSITZER KULTUR
}

\author{
Katarzyna Trybała-Zawiślak
}

DOI: https://doi.org/10.31577/szausav.2019.suppl.1.30

Keywords: Early Iron Age, Tarnobrzeg Lusatian culture, Thracian cultural circle, circle of forest-steppe cultures, vessels with diagonal and vertical plastic strips

\begin{abstract}
Vessels with diagonal and vertical plastic strips in the materials of the late phase of the Tarnobrzeg Lusatian culture

Pot-shaped vessels with specific plastic decoration in the form of diagonal or vertical strips are not common in the complexes from the late phase of the Tarnobrzeg Lusatian culture. An analogy with these finds is primarily provided by materials from the Thracian cultural circle. The elements of this style are also present in a much wider territory, because they are also registered in today's Slovakia or Ukraine. Some of them should be treated as local imitations of originals from the south. These vessels can also be treated as a manifestation of broadly understood contacts that took place in the Early Iron Age between various groups in this part of Central and Eastern Europe.
\end{abstract}

Die Tarnobrzeg Lausitzer Kultur gehört zu den am besten erforschten archäologischen Kultureinheiten, die in Südostpolen registriert werden. Nicht ohne Grund betrachtet man sie im Vergleich zu anderen Gruppen des Lausitzer Kulturkreises ebenfalls als eine der am besten erkannten Kultureinheiten, nicht nur in Bezug auf den Grad der Quellenbearbeitung, sondern auch im Hinblick auf zahlreiche analytische und synthetische Studien (Czopek 2009, 15). Das gilt insbesondere für die dritte Phase der Tarnobrzeg Lausitzer Kultur, die auf die Früheisenzeit fällt, d. h. sie umfasst die Zeitspanne vom Ende der Stufe Ha C, die Stufe Ha D und LT A. In der absoluten Chronologie sollte man diese Etappe zwischen die Mitte des 7. und das 4. Jh. v. Chr. setzen (Czopek 2007a, 192, 193; Trachsel 2004, 151, 152). In dieser Zeit haben wir sowohl mit einer Vergrößerung des Verbreitungsgebietes der Tarnobrzeg Lausitzer Kultur - neue Siedlungen erscheinen auch in den Zonen, die früher gar nicht besiedelt waren (Czopek 2008, 159) - als auch mit der Entstehung neuer Gräberfelder mit einer besonderen Innenstruktur (geordnete Grabkomplexe) zu tun (Trybała-Zawiślak 2015). Für diese Entwicklungsetappe der Lausitzer Kultur ist darüber hinaus eine größere als bisher Differenziertheit der Quellen typisch, was sich nicht nur auf die Metallfunde bezieht, sondern auch Glas-, Knochenartefakte und Keramik umfasst. Dieses Problem wurde schon mehrmals aufgegriffen, vor allem deshalb, weil es breite kulturelle Relationen betrifft, die als Treibkraft der in der Früheisenzeit im Südostpolen eingeleiteten Wandlungen betrachtet werden können (Czopek 2005, 249; 2007a; 2007b). In den Komplexen, die für die späte Phase der Tarnobrzeg Lausitzer Kultur charakteristisch sind, gibt es viele Elemente, die den lokalen Herstellern (vor allem den Keramikherstellern) fremd waren. Dabei sind mindestens einige, sowohl östliche, als auch südliche oder auch südöstliche Einflussrichtungen zu berücksichtigen. Man kann beispielsweise auf die Elemente hinweisen, die sich auf die Kulturen der Stempelkeramik zurückführen lassen (Gawlik/Przybyła 2005, 321-323). Nachweisbar sind ebenfalls die Einflüsse aus dem Milieu der Kuštanovice-Kultur (Czopek 2003, 231; Trybała-Zawiślak 2012, 155), wie auch die breit verstandenen östlichen Einflussnahmen, die die Waldsteppenzone Europas betreffen und sowohl mit dem vorskythischen Horizont als auch mit späteren Einflüssen aus dem skythischen Kulturkreis zusammenhängen (Czopek 2008, 163). Besonders deutlich ist das am Beispiel der Keramik erkennbar, vor allem im Falle der Schüsseln mit dem verdickten leicht einschwingenden Rand, wie auch der Topfgefäße mit plastischen Leisten und Löchern (manchmal sind sie nicht ganz durchgestochen) unter dem Mündungsrand. Derartige Keramikkomplexe wurden so charakteristisch oder sogar allgemein verbreitet, dass man sie als Erkennungszeichen für die Materialien der späten Phase der Tarnobrzeg Lausitzer Kultur betrachtet (Czopek 2003, 228). Im Folgenden wird diese zuletzt genannte 
Gefäßgruppe etwas näher behandelt, zumal sie ziemlich different ist. Hier lassen sich mindestens 3 Varianten unterscheiden. Die erste Variante bilden die Gefäße mit glatten Oberflächen, meistens braune und mit leicht S-förmigen Profilen. Sie sind entweder unverziert, oder besitzen plastische Knubben, Fingertupfen und Löcher unter dem Mündungsrand. Die zweite Variante umfasst zahlreiche Gefäße mit gerauten Oberflächen und ausgebauter plastischer Ornamentik in Gestalt von plastischen Leisten mit Fingertupfen- und Fingernagelverzierung, Knubben auf der ganzen Gefäßoberfläche und Löchern unter dem Mündungsrand. In dieser Gruppe finden sich sowohl schlanke als auch gedrungene beutelförmige Exemplare. Sowohl die erste als auch die zweite Variante sind aus zahlreichen Fundstellen der Tarnobrzeg Lausitzer Kultur, darunter aus sepulkralen und Siedlungsfundstellen, bekannt (vgl. Czopek 1991; 2004; 2014; Czopek u. a. 2014; Kostek 1991; Moskwa 1971; Szarek-Waszkowska 1975; Trybała-Zawiślak 2012). Die dritte Variante ist am spärlichsten vertreten, dabei aber im Kontext der behandelten Gefäßgruppe am interessantesten. Hierher gehören Töpfe mit einer, im Vergleich zu den oben beschriebenen Varianten, untypischen Verzierungsart. Die Ausergewöhnlichkeit dieser Verzierung bezieht sich jedoch nicht auf das formale Grundmerkmal, denn auch hier beobachtet man plastische Leisten mit Fingertupfenverzierung, sondern sie betrifft die Anordnung der Verzierungselemente auf der Gefäßoberfläche. Die Verzierungen kommen nämlich in schräger und (oder) senkrechter Anordnung auf der Gefäßoberfläche zum Vorschein. Das „,klassischste“ Beispiel derartiger Verzierung findet sich auf dem Gefäß aus Dębina, Fst. 6, Kr. Łańcut. Das ist eine multikulturelle Fundstelle mit deutlicher Dominanz der bronzezeitlichen und frühbronzezeitlichen Fundmaterialien. Diese Fundstelle ist auch bezüglich ihrer Funktion nicht homogen, denn man hat dort sowohl mit dem Gräberfeld der frühen Tarnobrzeg Lausitzer Kultur, als auch mit der in die späte Phase dieser Kultureinheit datierten Siedlung zu tun (Czopek/Trybała-Zawiślak 2014). Der behandelte Topf (Abb. 2: 3) kam im Siedlungskontext in einer der Gruben zusammen mit anderen Keramikfragmenten, u. a. einem anderen Topfgefäß vor (das Gefäß ist nicht vollständig erhalten geblieben, besitzt aber auch eine plastische Leiste unter dem Mündungsrand).

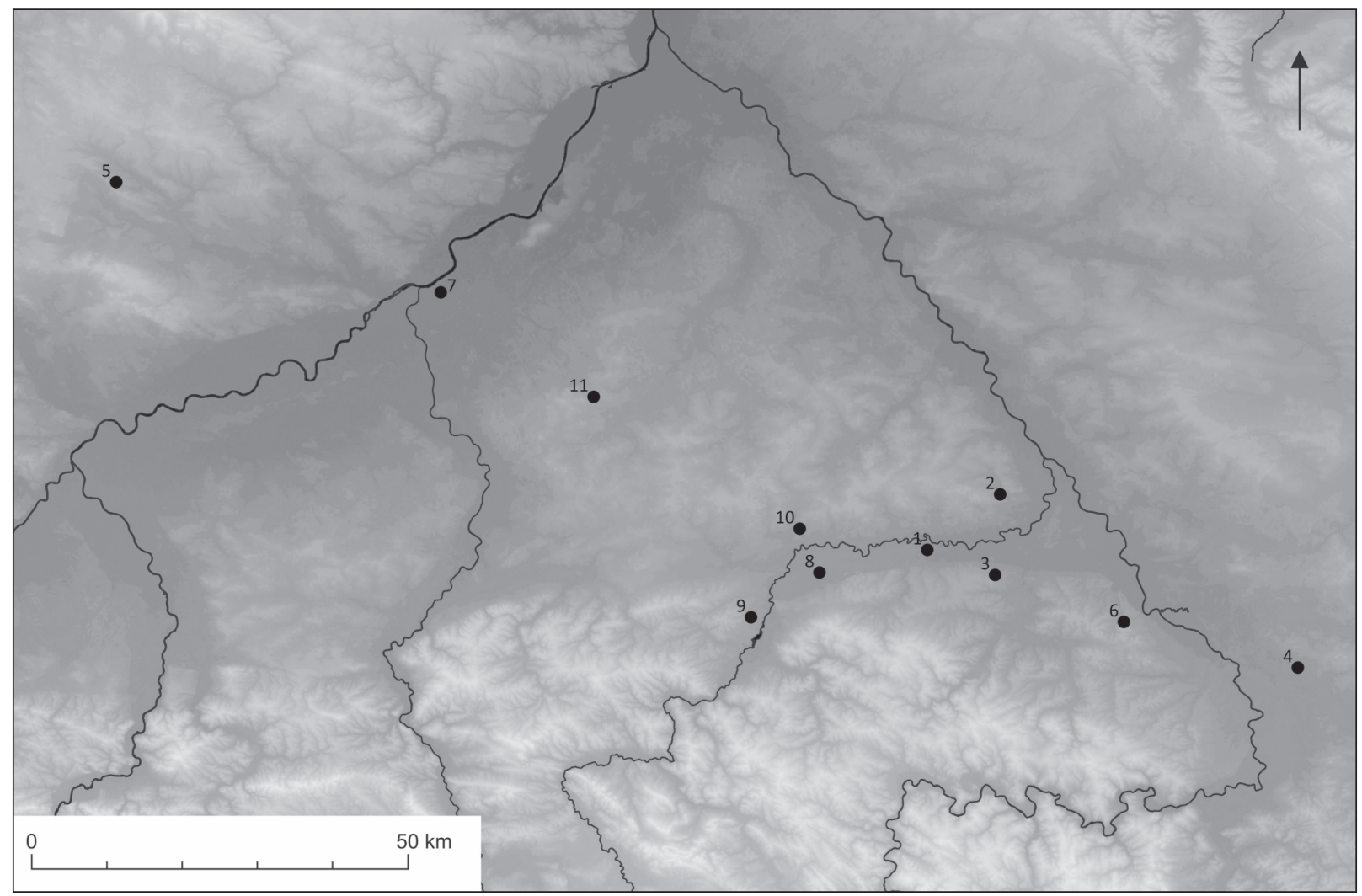

Abb.1. In Polen lokalisierte Fundstellen mit den im Text behandelten Keramikfunden (in alphabetischer Reihenfolge). 1 - Dębina, Kr. Łańcut; 2 - Grodzisko Dolne, Kr. Leżajsk; 3 - Grzęska, Kr. Przeworsk; 4 - Hruszowice, Kr. Przemyśl; 5 Janowice Poduszowskie-Antoniów, Kr. Busko; 6 - Jarosław, Kr. Jarosław; 7 - Krzemienica, Kr. Mielec; 8 - Łąka, Kr. Rzeszów; 9 - Rzeszów, Kr. Rzeszów; 10 - Stobierna, Kr. Rzeszów; 11 - Trzęsówka, Kr. Kolbuszowa. 
Die Gefäße mit schrägen und senkrechten plastischen Leisten besitzen nur wenige Analogien in den Materialien der Tarnobrzeg Lausitzer Kultur, doch es sind einige ähnliche Exemplare anzuführen. Allem Anschein nachweisen die Gefäße aus Łąka, Fst. 11-16, Kr. Rzeszów, ${ }^{1}$ und aus Grzęska, Fst. 1, Kr. Przeworsk, die größte Ähnlichkeit auf. Im ersten Fall haben wir mit einem ausgedehnten Komplex der Siedlungsfundstellen zu tun. In einem der Befunde der Tarnobrzeg Lausitzer Kultur wurden zwei Exemplare mit schrägen Leisten registriert, wobei eins der Gefäße eine Reihe waagerechter Knopfbuckel unter dem Mündungsrand, wie auch plastische Knubben, die kurze Segmente bildeten (auch in schräger Anordnung), besaß. Das zweite Gefäß hatte dagegen über den schrägen Leisten eine weitere waagerechte Leiste, die mit kurzen schräg gesetzten Einkerbungen verziert war. Auch hier befanden sich auf der Gefäßoberfläche Knopfbuckel (Abb. 2: 1, 2). Die zweite von den erwähnten Fundstellen ist ein früheisenzeitliches Gräberfeld der Tarnobrzeg Lausitzer Kultur. Hier wurde ein Topfgefäß mit einer waagerechten direkt unter dem Mündungsrand gesetzten Leiste und einer weiteren darunter auf dem Gefäßbauch gesetzten senkrechten Leiste geborgen. Interessant ist die Funktion des behandelten Gefäßes im sepulkralen Bereich. Seine Fragmente wurden absichtlich unter die Urnen aus zwei Gräbern gelegt (Czopek u. a. 2016, 78, Abb. 2: 15). Die Exemplare mit schrägen Leisten wurden ebenfalls an der Fundstelle Nr. 158 in Jarosław, Kr. Jarosław, registriert. Das ist eine große multikulturelle Siedlung mit dem deutlich wahrnehmbaren früheisenzeitlichen Horizont. Die erwähnten Töpfe wurden in zwei Befunden registriert. In einem von ihnen befand sich ein mit plastischen Leisten in schräger und senkrechter Anordnung verziertes Exemplar. In der Befundverfüllung registrierte man die Fragmente anderer Gefäße, darunter anderer Töpfe mit gerauten Oberflächen, einer profilierten Schüssel, eines Backtellers und eines Schöpfers. Im Falle des zweiten Befundes war der Fundkontext ähnlich, d. h. zusammen mit dem gerauten Topf, der ebenfalls mit schrägen Leisten verziert war, wurden noch ein Topfgefäß und Fragmente eines Backtellers gefunden (Czopek 2014, 83, 84, 147, Taf. XVI: 1; XIX: 3). Das Gefäß aus Hruszowice, Fst. 16, Kr. Przemyśl, bildet im Kontext der behandelten Fundmaterialien die neuste Entdeckung. Hruszowice ist eine ausgedehnte multikulturelle Siedlung, die im Vorfeld des Autobahnbaus exploriert wurde. In einem der Befunde stieß man auf einen großen Topf mit gerauter Oberfläche und mit der waagerechten plastischen Leiste direkt unter dem Mündungsrand, die zusätzlich mit Fingertupfen und Löchern verziert war, die nicht ganz von außen durchgestochenen waren. Darunter befanden sich schräge plastische Leisten mit analoger Fingertupfenverzierung (Abb. 2: 4). Auch die Fundstelle aus Rzeszów, Nr. 117, Kr. Rzeszów, kann in dem behandelten Kontext erwähnt werden. In mindestens zwei Befunden legte man dort Fragmente der Töpfe mit schräg gezogener plastischer Leistenverzierung frei (Czopek u. a. 2014, 179-181, Taf. XXIII: 1; XXV: 12). Es ist durchaus möglich, dass ähnliche Gefäße im Fundmaterial der Tarnobrzeg Lausitzer Kultur zahlreicher vorliegen, ihr fragmentarischer Erhaltungszustand lässt sie leider nicht identifizieren. Ihr Erhaltungszustand lässt auch Zweifel darüber aufkommen, ob sie der erwähnten Gefäßgruppe zugeordnet werden sollen. Ein gutes Beispiel ist hier die Siedlung in Stobierna, Fst. 2, Kr. Rzeszów. In einem der Befunde stieß man auf ein Gefäßfragment, das wahrscheinlich zu der hier behandelten Gefäßgruppe gehört, weil es neben der waagerechten auch eine schräge Leiste besitzt. Wie gesagt soll man diese Funde wegen des Erhaltungszustands der Gefäße nicht vorbehaltlos zuordnen (Ligoda/Stowik 2009, Taf. II: 3). Die oben genannten Funde machen jedoch im Kontext der behandelten Thematik nicht die ganze Quellenbasis aus, denn man kann noch auf andere Topffunde mit ähnlicher plastischer Verzierung hinweisen, die aus älteren Forschungen stammen und schon lange her veröffentlicht wurden. Zu nennen sind hier die Gräberfelder in Trzęsówka, Kr. Kolbuszowa (Moskwa 1971, Taf. XII: 15), und in Krzemienica, Kr. Mielec (Szarek-Waszkowska 1975, Taf. XXI: 9), wie auch die Siedlungsmaterialien aus Grodzisko Dolne, Kr. Leżajsk (Czopek 2007a), oder Janowice Poduszowskie-Antoniowa, Kr. Busko (Matoga 1989, Abb. 1-3). Die zuletzt genannte Fundstelle liegt außerhalb der geschlossenen Besiedlungszone der Tarnobrzeg Lausitzer Kultur (Abb. 1). Die Grundfrage lautet dementsprechend folgendermaßen: Soll man alle Gefäße mit schrägen und senkrechten Leisten genauso behandeln, d. h. als Ausdruck derselben fremden Kultureinflüsse, denn diese Interpretation soll zweifellos zuerst erwogen werden. Dieses Thema soll im Folgenden näher beleuchtet werden. Wie gesagt unterscheiden sich diese Gefäße von anderen für die späte Phase der Tarnobrzeg Lausitzer Kultur charakteristischen Materialien darin, dass sie keine lokalen Analogien besitzen. Diese sind eher im Süden im thrakischen Kulturkreis zu registrieren (Czopek 2014, 147), wo sehr ähnliche Exemplare vorliegen. Ihre Ornamentik ist sehr stark ausgebaut und umfasst waagerechte, senkrechte und schräge plastische Leisten mit Fingertupfen und manchmal mit ziemlich großen Knubben. Es kommt vor, dass sich die plastischen Leisten strahlenförmig von den

\footnotetext{
1 Ich möchte mich bei Herrn Mag. Mirosław Mazurek für die freundliche Bereitstellung der noch nicht veröffentlichten Materialien aus Łąka (Fundstelle 11-16) für die Zwecke dieses Beitrags bedanken.
} 
verschiedenförmigen Knubben abzweigen (Crişan 1969, Abb. 23; 24; 66; 75; Taf. XXXVIII: 3, 4; XLII: 12; XLIII: 3, 5, 8-10; LXXIV: 7; LXXIX: 11; Teodor/Nicu/Țau 2003, Abb. 6: 1; 12: 5). Unter den Funden der Tarnobrzeg Lausitzer Kultur bleiben identische Gefäße zwar aus, aber manche Exemplare knüpfen jedoch sehr stark an die oben angeführten Beispiele an. Die Gefäße aus Łaka sind ein sehr gutes Beispiel für die Verbindung vieler Verzierungsmotive. Der Topf aus Dębina repräsentiert ein klassisches Gefäß mit langen (sie verlaufen auf der ganzen Gefäßoberfläche) schrägen Leisten. Ein bisschen anders sollte man die Exemplare aus Hruszowice und Grzęska behandeln - hier hat man nämlich zusätzlich mit Löchern unter dem Mündungsrand zu tun, wobei sie nicht ganz durchgestochen sind. Derartiges Verzierungsmotiv ist für das skythische Waldsteppenmilieu sehr charakteristisch (Kovpanenko 1967, Abb. 24; 25; 37; 38) und plastische Leisten unter dem Mündungsrand sind auf fast jedem Topfgefäß zu treffen. Darüber hinaus kommen auch vereinzelt Exemplare mit einer kurzen senkrechten bzw. schrägen Leiste zum Vorschein (Kovpanenko 1967, Abb. 22: 3; 29: 3). Die Leiste mit kurzen Strichreihen, die auf einem in der Siedlung in Łaka gefundenen Gefäß, wie auch auf der Urne aus dem Gräberfeld in Krzemienica beobachtet wurde, ist im Bereich der Černoles-Kultur ziemlich verbreitet (Krušel'nic'ka 1998, Abb. 40: 3; 64: 1; 69: 1), wobei sie auch im thrakischen Kulturkreis vorkommt (Crişan 1969, Abb. 73, 7; 76: 1, 4; Taf. CVI: 2). Die Töpfe mit plastischen sowohl den waagerechten als auch schrägen Leisten sind auch aus der östlichen Slowakei bekannt. In der früheisenzeitlichen Siedlung in der Ortschaft Stretavka wurden ähnliche Formen registriert, obwohl die schrägen Leisten hier deutlich kürzer sind. Die Analogien fand man in der transkarpatischen Ukraine und genauer gesagt im Fundmaterial der Kuštanovice-Kultur (Miroššayová 1979, 130-132), wo solche Exemplare an den späthallstatischen Fundstellen in der Karpatenzone als massenhaft vorkommende Funde betrachtet werden (Popovič 2006, 535), wobei man in diesem Kontext eher auf ihre fassförmige Form als auf die Verzierungsart hinweist. Besonders interessant sind im Kontext der behandelten Keramik ebenfalls die Funde aus den Gebieten am unteren Lauf des Südlichen Bug (ukr. Boh), wo fast identische Gefäße in den Fundstelenkomplexen freigelegt wurden, die an das Ende des 6.-den Anfang des 5. Jh. v. Chr. datiert werden. Das sind kleine Töpfe mit charakteristischer Verzierung in Form von plastischen sowohl waagerechten als auch schrägen Leisten (einige Leisten zweigen sich strahlenförmig ab). Nicht unbedeutend ist auch ihre Herstellungstechnik. Es ist beachtenswert, dass diese Keramik aus Ton gefertigt wurde, der mit großen Schamottmengen gemagert wurde. Besonders bedeutend ist aber die Tatsache, dass ihre Analogien aus der karpatischen und donauländischen Zone stammen (Marčenko/Solovjov 1988). Wir haben demnächst also mit den gleichen Gefäßtypen und der gleichen Interpretation ihrer Abstammung zu tun, wie im Falle der Funde aus polnischen Gebieten.

Anscheinend kann man im Kontext der behandelten Keramik über die Verbindung unterschiedlicher Einflüsse aus unterschiedlichen Kulturkreisen sprechen, wobei auch die lokalen Traditionen und lokale Töpferei nicht unbedeutend sind. Die Gefäße mit schrägen und senkrechten Leisten erscheinen in der Tarnobrzeg Lausitzer Kultur vermutlich infolge der Kontakte mit den südlich bzw. südöstlich von ihrem Siedlungsgebiet wohnenden Gemeinschaften. Es fällt im Moment schwer, auf eine bestimmte Kultureinheit hinzuweisen. Man sollte eher ähnliche Tendenzen in der Keramikherstellung, oder auch die Tatsache in Betracht ziehen, dass diese Gemeinschaften nicht isoliert lebten und für Außenkontakte offen waren. Die entlehnten Muster konnten dann lokal modifiziert oder umgewandelt werden und vielleicht ist es deshalb so schwer, direkte Analogien zu finden. Jetzt soll noch auf die Chronologie der besprochenen Gefäße eingegangen werden. Im Süden werden sie je nach dem Typ zwischen 6.-5. Jh. v. Chr. oder 5.-4. Jh. v. Chr. datiert (Crişan 1969, 73-77). Im Falle der Funde aus der Waldsteppenzone vertreten die Töpfe mit plastischer Verzierung einen breiten chronologischen Horizont zwischen dem 7.-5. Jh. v. Chr. (Czopek u. a. 2016, 78). Das ändert nichts an der Tatsache, dass alle besprochnen Fundmaterialien, (sowohl diese, die auf die Tarnobrzeg Lausitzer Kultur bezogen werden als auch die den anderen Kultureinheiten zugeschriebenen Funde) den gleichen chronologischen Horizont vertreten und auf die Früheisenzeit, darunter eher auf ihre jüngere Etappe bezogen werden. Genaue chronologische Festlegung ist bei dem heutigen Forschungsstand unmöglich, vor allem ohne den Kontext der behandelten Funde zu analysieren. Dabei muss man die absolute Datierung, wie auch detaillierte Studien über die interkulturellen Beziehungen vor einem breiteren Hintergrund berücksichtigen.

Die Verzierung in Form von plastischen Leisten und Knubben oder Fingergrübchen (vor allem in Bezug auf die Topfgefäße) ist auf der früheisenzeitlichen Keramik allgemein verbreitet. Diese Bemerkung bezieht sich sowohl auf die Materialien der Tarnobrzeg Lausitzer Kultur als auch auf die aus derselben Zeit stammenden Fundstellen in der Ukraine (Krušel'nic'ka 1993; Marčenko/Solovjov 1988) oder in der Slowakei (Miroššayová 1994, 48, Abb. 10), wie auch weiter südlich auf den rumänischen und ungarischen 


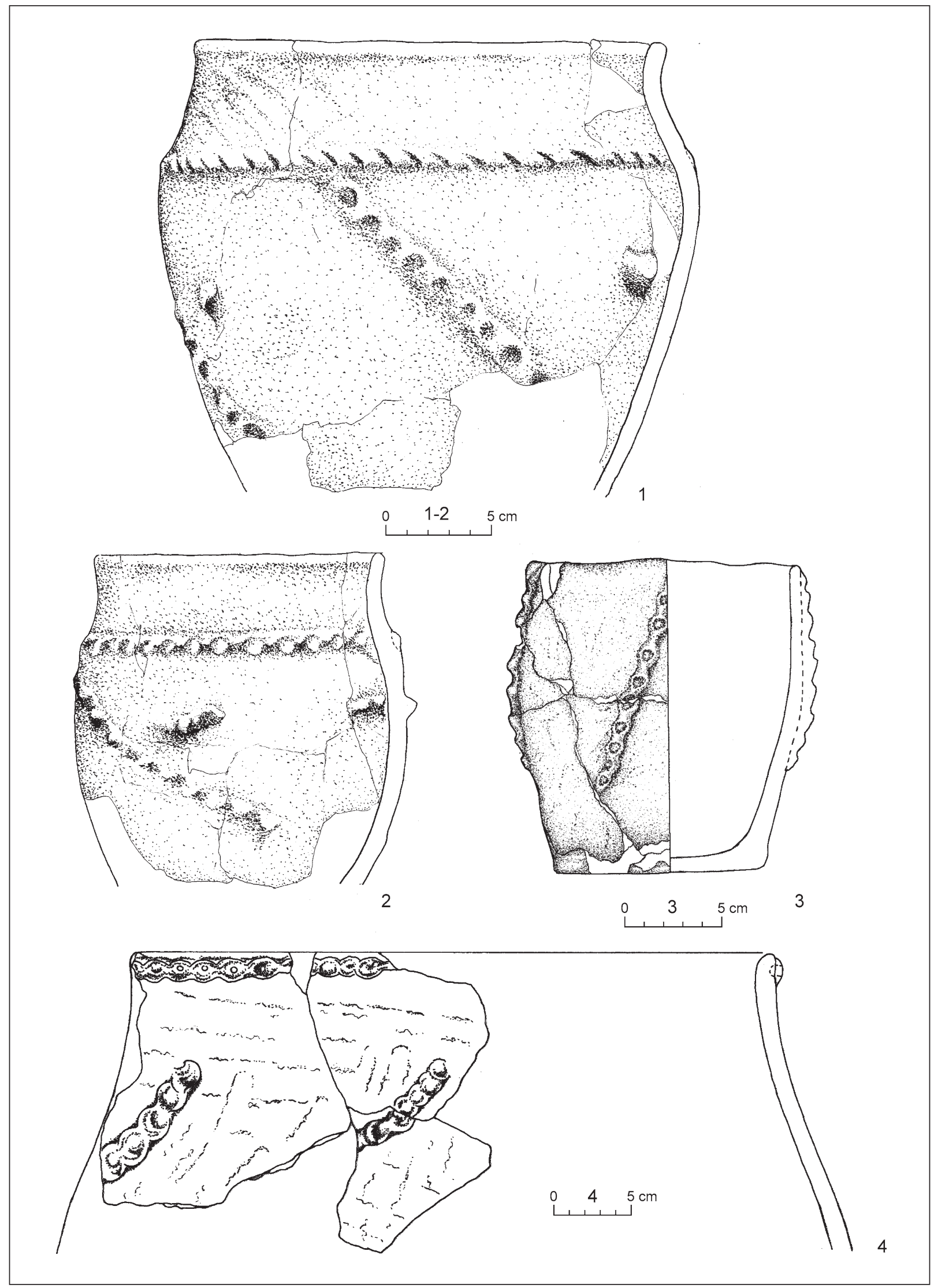

Abb. 2. Gefäße mit schrägen und senkrechten plastischen Leisten - Auswahl. 1, 2 - Łąka, Kr. Rzeszów (nach Drewniak u. a. 2012); 3 - Dębina, Kr. Łańcut (nach Jabłkowski 2013); 4 - Hruszowice, Kr. Przemyśl. 
Gebieten (Crişan 1969). Die behandelten Gefäße nehmen in diesen Komplexen einen besonderen Platz ein, da sie von weitgehenden kulturellen Kontakten zeugen. Im Falle der Tarnobrzeg Lausitzer Kultur gehören sie zu den wichtigen Elementen, die ihr außergewöhnliches Bild in der Früheisenzeit prägten.

\section{LITERATUR}

Crişan 1969

Czopek 1991

Czopek 2003

Czopek 2004

Czopek 2005

Czopek 2009

Czopek 2007a

Czopek 2007b

Czopek 2008

Czopek 2014

Czopek u. a. 2014

Czopek u. a. 2016

Czopek/Trybała-Zawiślak 2014

Drewniak u. a. 2012

Gawlik/Przybyła 2005

Jabłkowski 2013

Kostek 1991

Kovpanenko 1967

Krušel'nic'ka 1993
I. H. Crişan: Ceramica Daco-Getica. Cu specialăprivire la Transilvania. Bucureşti 1969.

S. Czopek: Sprawozdanie z badań wykopaliskowych w Białobrzegach, woj. Rzeszów (Fundstellen 1 und 5) aus den Jahren 1982-1984. Materiały i Sprawozdania Rzeszowskiego Ośrodka Archeologicznego 1980-1984, 1991, 165-172.

S. Czopek: Między Południem a Wschodem - importy i naśladownictwa ceramiki w materiałach grupy tarnobrzeskiej. In: J. Gancarski (Hrsg.): Epoka brazu $i$ wczesna epoka żelaza w Karpatach polskich. Materiaty z konferencji. Krosno 2003, 215-238.

S. Czopek: Cmentarzysko ciałopalne z wczesnej epoki żelaza w Knapach. Rzeszów 2004.

S. Czopek: Epoka brązu i wczesna epoka żelaza w Kotlinie Sandomieskiej uwagi o stanie badań i aktualnych problemach badawczych, In: M. Kuraś (Hrsg.): Archeologia Kotliny Sandomierskiej. Rocznik Muzeum Regionalnego w Stalowej Woli 4. Stalowa Wola 2005, 241-254.

S. Czopek: Aktualne problemy w badaniach tarnobrzeskiej kultury łużyckiej, In: S. Czopek/K. Trybała-Zawiślak (Hrsg.): Tarnobrzeska kultura łużycka - źródta $i$ interpretacje. Rzeszów 2009, 15-31.

S. Czopek: Grodzisko Dolne. Stanowisko 22 - wielokulturowe stanowisko nad dolnym Wisłokiem, cz. 1. Od epoki kamienia do wczesnej epoki żelaza. Rzeszów 2007.

S. Czopek: Środkowoeuropejska rubież kulturowa - między wschodem a zachodem w epoce brązu i wczesnej epoce żelaza. In: M. Dębiec/M. Wołoszyn (Hrsg.): U źródeł Europy Środkowo-Wschodniej: pogranicze polsko-ukraińskie z perspektywy badań archeologicznych. Collectio Archaeologica Ressoviensis 5. Rzeszów 2007, 109-125.

S. Czopek: Uwagi o sytuacji kulturowej we wczesnej epoce żelaza na terenie południowo-wschodniej Polski. In: M. Mogielnicka-Urban (Hrsg.): Opera ex aere: Studia z epoki brazu i wczesnej epoki żelaza dedykowane profesorowi Janowi Dąbrowskiemu przez przyjaciót, uczniów i kolegów z okazji siedemdziesięciolecia urodzin. Warszawa 2008, 159-167.

S. Czopek: Stanowisko 158 w Jarosławiu, woj. podkarpackie II. Osadnictwo od starszej epoki brazu do okresu rzymskiego. Via Archaeologica Ressoviensia 4. Rzeszów 2014.

S. Czopek/D. Niemasik/W. Pasterkiewicz/A. Pelisiak: Rzeszów, stanowisko 117 osada wielokulturowa. Rzeszów 2014.

S. Czopek/J. Ligoda/J. Podgórska-Czopek/J. Rogóż: Cmentarzysko tarnobrzeskiej kultury tużyckiej z wczesnej epoki żelaza w Grzessce, pow. Przeworski. Rzeszów 2016. S. Czopek/K. Trybała-Zawiślak: Wybrane aspekty obrządku pogrzebowego we wczesnej fazie tarnobrzeskiej kultury łużyckiej na przykładzie stan. 6 w Dębinie, pow. Łańcucki. Haykobi ctydii 7, 2014, 118-130.

K. Drewniak/A. Mazurek/M. Mazurek/A. Nowak/A. Okoniewska/J. Tarchała/S. Woźniak: Opracowanie ratowniczych badań wykopaliskowych na stanowisku 11-16 (A4/68-73) w Łace, gm. Trzebownisko. Rzeszów 2012. Unveröffentlichte Arbeit.

A. Gawlik/M. Przybyła: Początki wczesnej epoki żelaza w Kotlinie Sandomierskiej. In: M. Kuraś (Hrsg): Archeologia Kotliny Sandomierskiej. Rocznik Muzeum Regionalnego w Stalowej Woli 4. Stalowa Wola 2005, 313-352.

M. Jabłkowski: Obiekty 101, 109 i 110 z wczesnej epoki żelaza ze stanowiska nr 6 w Dębinie. Rzeszów 2013. Unveröffentlichte Arbeit.

A. Kostek: Cmentarzysko grupy tarnobrzeskiej w Wietlinie III, gm. Laszki, woj. Przemyśl. Materiały i Sprawozdania Rzeszowskiego Ośrodka Archeologicznego 19801984, 1991, 5-44.

G. T. Kovpanenko: Plemena skifs'kogo času na Vorsklí. Kï̈ 2012.

L. I. Krušel'nic'ka: Kuštanovic'ka grupa pam'jatok. In: Pam'jatki gal'štatskogo peridu v mejričči Visly, Dnistra i Prip'jati. Kiev 1993, 250-286. 
Krušel'nic'ka 1998

Ligoda/Stowik 2009

Marčenko/Solovjov 1988

Matoga 1989

Miroššayová 1979

Miroššayová 1994

Moskwa 1971

Popovič 2006

Szarek-Waszkowska 1975

Teodor/Nicu/Tau 2003

Trachsel 2004

Trybała-Zawiślak 2012

Trybała-Zawiślak 2015
L. I. Krušel'nic'ka: Čornolis'ka kul'tura seredn'ogo Pridnìstrov'â. L'vìv 1998.

J. Ligoda/K. Słowik: Wstępne wyniki badań archeologicznych przeprovadzonych na stanowisku 2 w Stobiernej, pow. Rzeszów (badania na trasie drogi ekspresowej S-19). In: S. Czopek/K. Trybała-Zawiślak (red.): Tarnobrzeska kultura łużycka-źródła i interpretacje. Collectio Archaeologica Ressoviensis XI. Rzeszów 2009, 353-363.

K. K. Marčenko/S. L. Solovjov: Nova grupa lìpnoï keramiki Nižnogo Pobužžâ pìzn'oarhaïčnogo času. Arheologiâ 63, 1988, 56-59.

A. Matoga: Z badań nad młodszą fazą grupy tarnobrzeskiej kultury łużyckiej na Kielecczyźnie. In: A. Barłowska/E. Szałapata (Hrsg): Grupa tarnobrzeska kultury tużyckiej. Materiały z konferencji 12-14 listopada 1986 w Rzeszowie. Rzeszów 1989, 263-284.

E. Miroššayová: Záchranný výskum halštatského sídliska v Stretavke, okr. Michalovce. Archeologické rozhledy 31, 1979, 121-143.

E. Miroššayová: Sídlisko z neskorej doby halštatskej v Čečejovciach. Slovenská archeológia 62, 1994, 37-68.

K. Moskwa: Późnołużyckie cmentarzysko w Trzęsówce, pow. Kolbuszowa. Materiały i Sprawozdania Rzeszowskiego Ośrodka Archeologicznego 1967, 1971, 9-98. I. Popovič: Zakarpatti za dobi rannogo zaliza. Kraków - Lwów 2006.

E. Szarek-Waszkowska: Cmentarzysko kultury łużyckiej w Krzemienicy, pow. Mielec. Materiały i Sprawozdania Rzeszowskiego Ośrodka Archeologicznego 1970_ 1972, 1975, 3-51.

S. Teodor/M. Nicu/S. Țau: Ceramica din aşezarea geto-dacică de la Poiana, județul Galați. Archeologia Moldovei 23-24, 2003, 21-182.

M. Trachsel: Untersuchungen zur relativen und absoluten Chronologie der Hallstattzeit. Universitätsforschungen zur prähistorischen Archäologie 104. Bonn 2004.

K. Trybała-Zawiślak: Kłyżów stan. 2 i Mokrzyszów 2. Cmentarzyska ciałopalne z wczesnej epoki żelaza. Rzeszów 2012.

K. Trybała-Zawiślak: Przestrzeń i czas - cmentarzyska tarnobrzeskiej kultury tużyckiej w kontekście rozplanowania grobów. Rzeszów 2015.

\title{
Vessels with diagonal and vertical plastic strips in the materials of the late phase of the Tarnobrzeg Lusatian culture
}

\author{
Katarzyna Trybała-Zawiślak
}

Summary

The Tarnobrzeg Lusatian culture is one of the best recognized archaeological units identified in south-eastern Poland. Particularly, this applies to its third phase falling into the Early Iron Age, and thus covering the final part of the Ha C period, the Ha D and Lt A periods. Considering absolute chronology this stage should be placed in the range of the mid- $7^{\text {th }}$ and the $4^{\text {th }}$ century $\mathrm{BC}$. One of the most characteristic evidence identified at sites from that time are vessels with plastic ornaments. The least numerous group are vessels decorated with oblique and vertical strips with fingerprints. An analogy with these finds should be sought in the south, i.e. in the Thracian cultural circle, where they are dated from the $6^{\text {th }}$ to $4^{\text {th }}$ century BC. However, similar items are identified in a much wider territory, among others in eastern Slovakia, where Transcarpathian analogies are indicated for them. Similar vessels, although more in a morphological dimension than ornamental patters, are known from the Kushtanovice culture, and as for the Carpathian zone their widespread occurrence is emphasized. It is also possible to indicate certain similarities of some vessels known from the broadly understood forest-steppe zone. Almost identical items were also recorded at the sites dated at the end of the $6^{\text {th }}$-beginning of the $5^{\text {th }}$ century BC from the areas of the lower Pobuże, where they are also interpreted as a manifestation of influences from the south. With regard to the current stage of research, it is rather impossible to indicate a particular cultural group in the context of analogy to the aforementioned vessels. Probably we are also dealing with local transformation of designs borrowed from the south. However, it is worth considering that we have registered very similar trends in ceramic ware of communities living in quite remote areas. Therefore, it demonstrates their openness to external contacts, and in the case of the Tarnobrzeg Lusatian culture it is one of the important elements characterizing its shape in the Early Iron Age. 
Fig. 1. Sites from Polish territory with the discussed artefacts in the text (in alphabetical order): 1 - Dębina, Łańcut district; 2 - Grodzisko Dolne, Leżajsk district; 3 - Grzęska, Przeworsk district; 4 - Hruszowice, Przemyśl district; 5 - Janowice Poduszowskie-Antoniów, Busko district; 6 - Jarosław, Jarosław district; 7 - Krzemienica, Mielec district; 8 - Łaka, Rzeszów district; 9 - Rzeszów, Rzeszów district; Stobierna, Rzeszów district; 11 - Trzęsówka, Kolbuszowa district.

Fig. 2. Selected examples of vessels with diagonal and vertical strips: 1, 2 - Łąka, Rzeszów district (after Drewniak u. a. 2012); 3 - Dębina, Łańcut district (after Jabłkowski 2013); 4 - Hruszowice, Przemyśl district.

Übersetzung Barbara Jachym Translated by Beata Kizowska-Lepiejza

Dr Katarzyna Trybała-Zawiślak

Instytut Archeologii

Uniwersytet Rzeszowski

ul. Moniuszki 10

PL - 35-015 Rzeszów

katarzyna.trybala@archeologia.rzeszow.pl 